\title{
Cell-Based Strategies for the Treatment of Kidney Dysfunction: A Review
}

\author{
Christopher J. Pino ${ }^{\mathrm{a}}$ Alexander S. Yevzlin $^{\mathrm{d}}$ James Tumlin ${ }^{\mathrm{e}}$ H. David Humes ${ }^{\mathrm{a}-\mathrm{c}}$ \\ ${ }^{a}$ Innovative BioTherapies, Inc., ${ }^{b}$ Department of Internal Medicine, University of Michigan Medical School, and \\ ${ }^{c}$ CytoPherx, Inc., Ann Arbor, Mich., d Division of Nephrology, Department of Medicine, University of Wisconsin \\ School of Medicine and Public Health, Madison, Wisc., and ' University of Tennessee College of Medicine \\ Chattanooga, Chattanooga, Tenn., USA
}

\section{Key Words}

Stem cell $\cdot$ Renal tubule assist device $\cdot$ Renal replacement • Acute kidney injury $\cdot$ End-stage renal disease $\cdot$ Cell-based therapy $\cdot$ Tissue engineering

\begin{abstract}
Conventional treatment of acute and chronic renal diseases has focused on solute removal. Novel strategies aim to treat the multifactorial disease states of acute kidney injury and chronic kidney disease by mitigating inflammation. Cellbased technologies for the treatment of kidney dysfunction fall under two broad categories: cell therapy and cell processing. Cell therapy utilizes cells that are isolated, cultured outside of the body, and reintroduced as therapy, leveraging beneficial metabolic and synthetic functions. For example, renal tubule cells have been used to provide gluconeogenesis, ammoniagenesis, metabolism of glutathione, catabolism of important peptide hormones, growth factors, and cytokines critical to multiorgan homeostasis and immunomodulation to treat renal dysfunction. Cell processing focuses on altering the characteristics of cell populations inside the body to provide therapy. The selective cytopheretic device is an example of this novel therapeutic strategy that aims to modulate the innate immune response during organ dysfunction, additional organ injury, by binding and deacti-
\end{abstract}

vating leukocytes. In this review, both cell therapy and cell processing approaches will be discussed in the context of acute kidney injury and chronic renal disease.

Copyright $\odot 2012$ S. Karger AG, Basel

\section{Introduction}

Acute kidney injury (AKI) affects up to 200,000 people in the United States annually, with a mortality rate of around 50\% [1-3]. AKI develops predominantly due to the injury and necrosis of renal proximal tubule cells as a result of ischemic or toxic insult [4]. The cause of death subsequent to AKI is generally the development of systemic inflammatory response syndrome, frequently secondary to bacterial infection or sepsis, resulting in cardiovascular collapse and ischemic damage to vital organs, culminating in multiorgan failure (MOF) [5].

Similarly, the morbidity and mortality rates associated with end-stage renal disease (ESRD) remain high. In 2007, expenditure on ESRD in the United States was USD 23.9 billion, accounting for $5.8 \%$ of the total Medicare budget [6]. This included an incidence of approximately 360 new patients with ESRD per million people, and an overall prevalence of almost 1,700 patients with ESRD per million Americans.

\section{KARGER \\ Fax +4161306 1234}

E-Mail karger@karger.ch

www.karger.com
(C) 2012 S. Karger AG, Basel

0253-5068/12/0342-0117\$38.00/0

Accessible online at:

www.karger.com/bpu
Dr. David Humes

Innovative BioTherapies, Inc.

401 W. Morgan Rd.

Ann Arbor, MI 48108 (USA)

Tel. +1 734213 8350, E-Mail dhumes@innbio.com 
In both AKI and ESRD, despite decades of improvements in the provision of renal replacement therapy, the morbidity and mortality rates associated with these disease states have remained largely unaltered. Conventional renal replacement therapies treat volume overload, uremia, acidosis, and electrolyte derangements but have no direct effect on the immune dysregulation that frequently attends AKI and ESRD [7]. Inflammatory cascades initiated by endothelial dysfunction in systemic inflammatory response syndrome are further dysregulated in the setting of AKI, as suggested by recent data that demonstrate that the levels of the proinflammatory cytokines IL- 6 and IL-8 in the plasma predict mortality in patients with AKI $[8,9]$. Furthermore, strategies that modulate the inflammatory response provide significant beneficial effects in experimental AKI [10].

Thus, improved therapeutic devices have to be developed with the capacity to replace a wider range of kidney functions, thereby reducing morbidity, mortality, and the overall economic impact associated with AKI and ESRD. The purpose of this review is to describe promising cellbased approaches to mitigating the inflammation that accompanies acute and chronic kidney dysfunction [11]. Cell-based approaches in this review will focus on two broad categories: cell therapy and cell processing. Cell therapy relies on isolated cell populations that are cultured outside of the body and reintroduced during therapy, whereas the cell processing approach seeks to alter a cell population inside the body.

\section{Cell Therapy}

To date, the treatment of acute and chronic kidney diseases by cell therapy has been dependent on the development of robust stem cell sources. Stem cells are characterized by their capacity for self-renewal and ability to differentiate into specialized cell types. For an in-depth treatment of the stem cells used for renal applications, see Pino and Humes [12]. Also see reviews on putative resident progenitor/stem cells of the kidney [13-16].

In brief, a number of different stem cell sources have been explored for renal disease applications, including embryonic stem cells, induced pluripotent stem cells, and adult stem cells, either from mesenchymal stem cell or renal epithelial progenitor sources. Embryonic stem cell and induced pluripotent stem cell approaches currently have substantive safety and regulatory issues not completely understood or evaluated. Therefore, in this review, cell-based therapies for the treatment of renal disease will focus on adult stem cell treatments. The two main modes of administration for cell-based therapy are direct injection and tissue engineering approaches. Direct injection relies on the inherent capabilities of stem cells to differentiate, organize, and integrate into existing tissues to restore function. Tissue engineering approaches are based on in vitro differentiation of stem cells on biomaterial scaffolds, which are then applied in vivo or ex vivo to provide therapy. This will be the focus of this review.

\section{Tissue Engineering Approach to Renal Progenitor Therapies}

Tissue engineering involves the in vitro manipulation of cells applied to biomaterials, which may be biodegradable or permanent substrates for cell attachment, to produce a device for implantation or incorporation into an extracorporeal circuit. Cell therapy with a single differentiated cell type to replace a specific metabolic or catabolic function is currently in practice [12]. However, the biotechnology tools required to fabricate a complete, functioning organ for transplantation are still in their infancy.

The strategy adopted by Humes and colleagues $[8,17]$ has been to administer cell therapy from an extracorporeal circuit, allowing for immunoisolation of a cell device, eliminating immunorejection issues, and enabling the use of allogeneic cells. In brief, this strategy utilized hemofiltration as a working substitute for glomerular filtration, with metabolic and synthetic functions of proximal tubule cells replaced through the application of a renal tubule assist device (RAD). The RAD consisted of primary renal cells, isolated and expanded from adult kidneys in vitro $[18,19]$, that were seeded on the inner surface of a hemofilter with polysulfone hollow fibers $[17,20$, 21]. Cell attachment was promoted by collagen type IV [22]. In vitro testing of the RAD demonstrated differentiated renal tubule cell function including active transport, renal cell-specific metabolic activity, and endocrine secretion [22].

In ex vivo animal testing, RADs containing either porcine or human cells were evaluated on uremic dogs following bilateral nephrectomy. Improvements in multiple physiological parameters were observed in RADtreated animals compared to acellular RAD controls $[22,23]$. Furthermore, in canine and porcine models of AKI with septic shock, RAD treatment was shown to modulate plasma cytokine levels, maintain better car- 
diovascular performance, and increase survival times $[22,24]$.

Promising preclinical studies prompted an initial phase I/II FDA clinical trial of RAD treatment of 10 critically ill patients with AKI and MOF receiving continuous venovenous hemofiltration [25]. The results from this study demonstrated that RAD therapy was safe for use for up to $24 \mathrm{~h}$ and that the cell device retains viability, durability, and functionality throughout therapy [25]. In a phase II randomized, controlled, open-label trial, involving 58 patients with $\mathrm{AKI}$ at 12 clinical sites, RAD treatment promoted a statistically significant survival advantage (33\% mortality at day 28 ) over patients treated by conventional continuous venovenous hemofiltration (61\% mortality at day 28) [26].

During a follow-up phase IIb study to evaluate a commercial manufacturing process for the RAD, the clinical study was suspended after an interim analysis revealed an unanticipated high survival rate of patients treated with sham control RAD without cells [27]. These findings with the RAD led to 2 separate therapy approaches: one device approach without cells called the selective cytopheretic device (SCD) (discussed in further detail below, under Cell Processing Approach), and a new cell-based device called the bioartificial renal epithelial cell system (BRECS), designed from the insight provided by the phase IIb study. The phase IIb study exposed the fact that production and distribution of RADs would be a major obstacle in the widespread adoption of renal cell therapy. In response, an engineered solution was initiated through the development of a cell system that would be cryopreservable, to enable distribution, storage, and therapeutic use at point-of-care facilities. Cryopreservation of biological samples and tissue engineering constructs have been widely recognized as an obstacle to the practical implementation of regenerative medicine [28]. The BRECS was developed with the intent to be cryopreserved; a device which functions as a combined bioreactor, cryostorage device, and cell therapy delivery system. Briefly, porous, niobium-coated carbon disks were used as cell scaffolds within the BRECS, and culture media was perfused through and around the porous disks. A recently developed technique of expanded propagation allowed for the amplification of kidney progenitor cells from human kidney tissue procured from National Disease Research Interchange (Philadelphia, Pa., USA), to serve as a robust therapeutic cell source for seeding BRECS. Application of the defined expanded propagation method resulted in an increase of up to 8 orders of magnitude in cell yield over historic, standard propagation techniques [29]. In vitro measurements of glucose and oxygen consumption, lactate generation, and glutathione degradation suggest the maintenance of more than $1 \times 10^{8}$ cells in each BRECS during up to 5 months in culture [30]. Ex vivo large animal studies suggest that utilizing the BRECS in conjunction with standard hemofiltration is a promising approach to treat both AKI and ESRD [30-32].

\section{Cell Processing Approach}

In a retrospective analysis of the phase IIb RAD study, paying particular attention to sham control RAD groups, the improved survival rate for acellular RAD treatment was demonstrated only when regional citrate anticoagulation was utilized in additional to systemic heparinization, and not when only systemic heparin anticoagulation was used. Patients treated with the acellular RAD had a mortality rate of $50 \%$ if treated with heparin versus $25 \%$ if treated with citrate ( $\mathrm{n}=12$ for each treatment $\mathrm{arm})$ at 28 days and 75 versus $33 \%$ at 90 days $\left(\chi^{2}<0.05\right)$ [27]. The subgroups were comparable with respect to sequential organ failure assessment scores, organ failure number, and incidence of sepsis.

Subsequent studies focused on understanding the mechanism of action by this noncellular, RAD hemofilter during regional citrate anticoagulation. It was hypothesized that the observed improvement in patient survival was mediated by a stabilizing effect that the acellular filter had, specifically conferred in a low-ionized calcium environment created by citrate administration in the blood circuit [33, 34]. Immunofluorescence microscopy of the sham cartridges after patient treatment demonstrated adherent leukocytes on the outer surface of the membranes of the cartridge along the blood flow path within the extracorporeal circuit [27]. The sequestered leukocytes were dominated by neutrophils. The ability of leukocytes to bind to the outer walls of the hollow fiber membranes rather than the inner walls, which is the conventional blood flow path, was recognized to be due to the low shear forces of blood flow. The shear stress of blood along the outer wall of the membrane was near capillary shear stress of $<1 \mathrm{dyn} / \mathrm{cm}^{2}$ compared to the shear stress of nearly $100 \mathrm{dyn} / \mathrm{cm}^{2}$ of blood when flowing along the inner conventional surfaces of the hollow fiber membranes. The role of citrate infusion in this device related to the effect of citrate to lower the ionized calcium levels of blood to below $0.4 \mathrm{~mm}$, a level which inhibits the coagulation system of blood. This lower ionized calcium level also has an inhibitory effect on neutrophil activation 
Fig. 1. SCD-CRRT circuit diagram depicting regional citrate anticoagulation. Citrate is administered before SCD, and ionized calcium is replaced after SCD, prior to blood return to the patient.

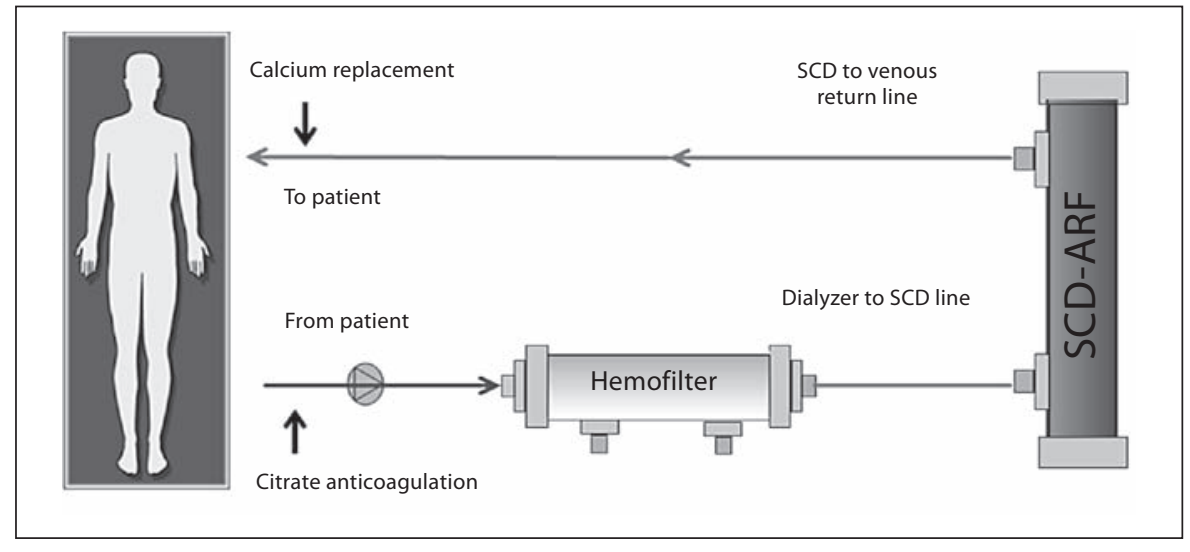

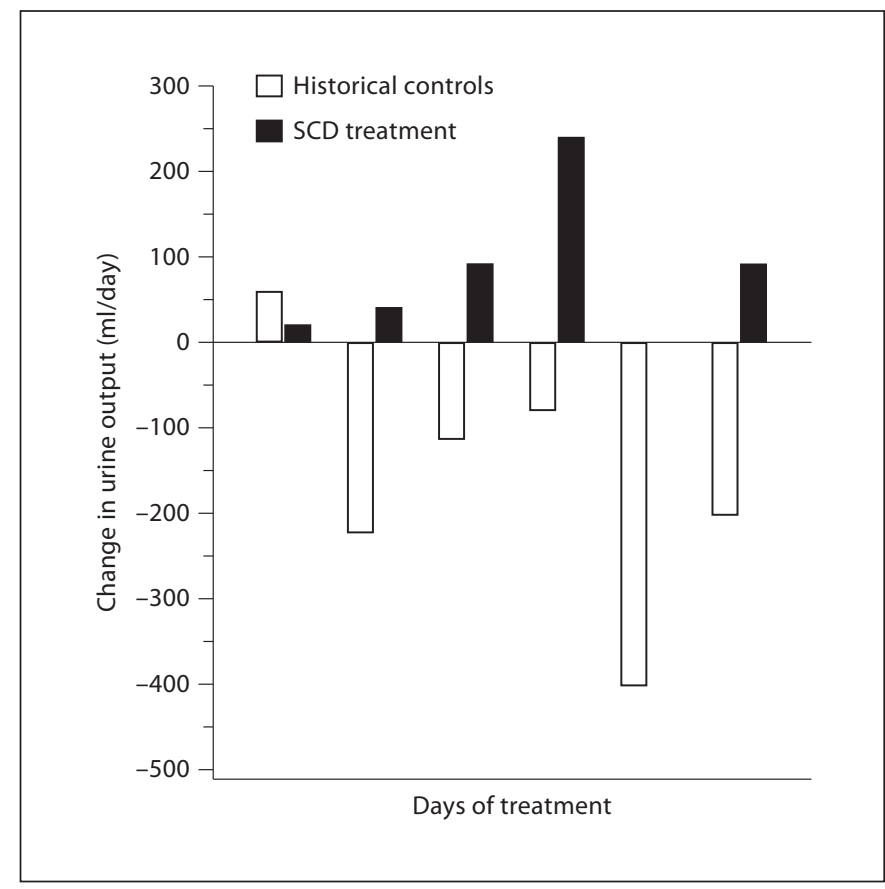

Fig. 2. Change in mean urine output versus time in a pilot study of SCD-treated patients (black bars) compared to the PICARD database case-matched historical controls (white bars).

[35], resulting in a simultaneous combination effect to sequester activated circulating leukocytes and alter the activity of the bound leukocytes. Further studies now suggest that the bound leukocytes may be subsequently released back to the systemic circulation in an altered apoptotic state. Consequently, the membrane cartridge is referred to as an SCD due to its ability to sequester cells, and in the presence of citrate anticoagulation, the SCD is an immunomodulatory device.

Blood Purif 2012;34:117-123
The SCD was further tested in a septic shock porcine model where SCD efficacy was evaluated treating AKI associated with septic shock caused by administering Escherichia coli into the peritoneum of pigs [24]. This septic shock model demonstrated that the SCD treatment lowers neutrophil activity (serum myeloperoxidase and CD11b cell surface expression), diminishes neutrophil tissue invasion, decreases systemic capillary leak, preserves cardiac output and mean arterial pressure, and prolongs survival time [35]. The SCD was also tested in a bovine model of cardiopulmonary bypass, and similarly showed decreased numbers of leukocytes throughout SCD therapy [36]. Further experiments have suggested that the 'catch and release' of activated neutrophils within the SCD [35] promoted the activated neutrophil to enter a delayed apoptotic state. This observation is consistent with previous work that demonstrated that blocking calcium entry into a neutrophil activates the apoptotic pathway to programmed cell death [37].

With these favorable animal studies confirming the early mechanistic observations from the RAD study, the evaluation of the SCD with citrate anticoagulation has continued in two exploratory pilot clinical trials in intensive care unit (ICU) patients with AKI and MOF. This patient group was chosen due to the ease of incorporating this device into the standard continuous renal replacement therapy (CRRT) blood circuit during treatment of these critically ill patients (fig. 1). These early exploratory clinical trials have demonstrated an excellent safety profile and compelling efficacy impact [27, 38, 39]. Leukopenia and sustained thrombocytopenia were not observed in these clinical studies. Accelerated renal recovery with CRRT discontinuation and an approximately $50 \%$ or greater relative improvement in survival rates have been observed. 
Fig. 3. SCD pilot trial versus historical outcome data in AKI in the ICU.

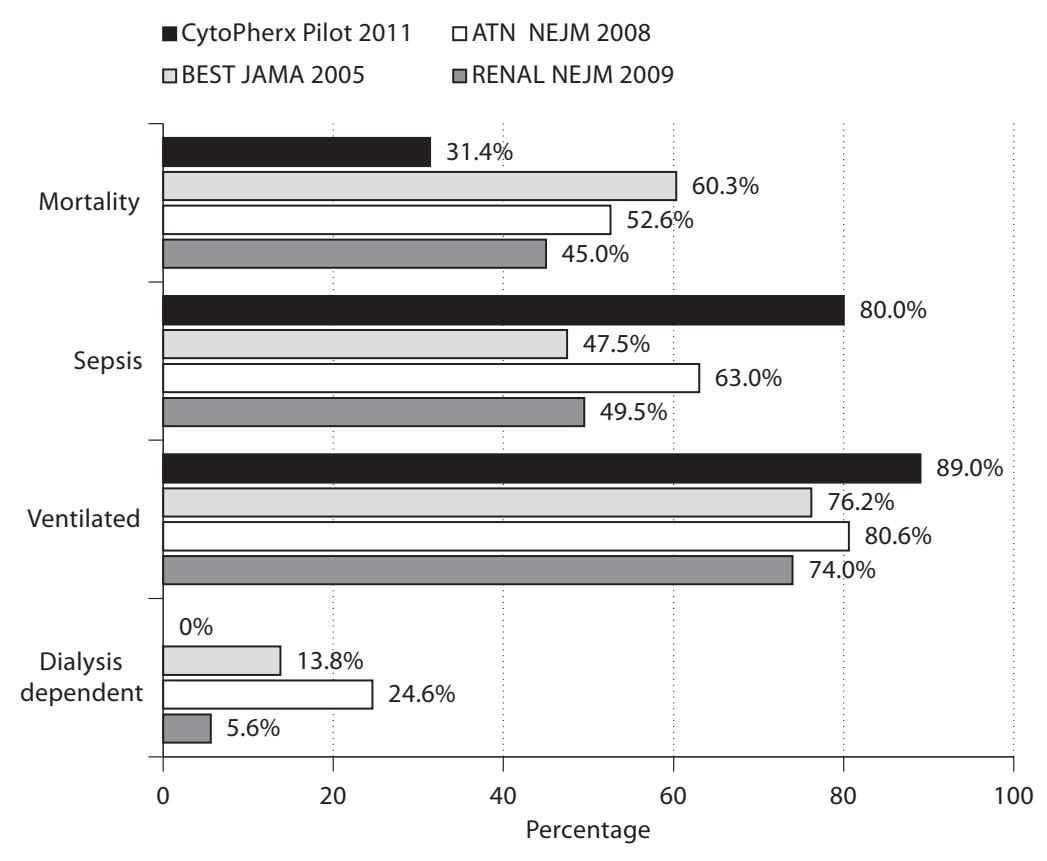

A prospective, single-arm, single-center clinical study was conducted to evaluate the safety and efficacy of the SCD in a dose-ranging study in ICU patients with AKI and MOF [38]. This study was approved by the local institutional review board as a nonsignificant risk designation. The dose-ranging study included increasing the effective outer membrane surface area from 1.0 to $1.4 \mathrm{~m}^{2}$ and by increasing SCD treatment from $72 \mathrm{~h}$ up to 7 days with SCD replacement every $24 \mathrm{~h}$. Nine patients were enrolled in the trial and were compared with historical casematched controls with respect to age and sequential organ failure assessment score utilizing the PICARD/NIH database [40]. The mortality rate for the case-matched controls was $78 \%$, while the mortality in the SCD treatment group was $22 \%(\mathrm{p}=0.027)$. Multiple regression analysis identified treatment with SCD as the only significant variable affecting mortality. Mean total urine output in the patients receiving SCD treatment increased from a baseline of approximately $500 \mathrm{ml} /$ day to more than $2,000 \mathrm{ml} /$ day by day 7 of treatment as opposed to historical case-matched controls, whose urine output decreased with time (fig. 2). In fact, in the last 5 patients receiving the larger SCD membrane renal function and urine output were improved after $72 \mathrm{~h}$ so that no dialytic treatment was necessary after 3 days. Once again, treatment with SCD was well tolerated, without significant effects on hematological parameters, and with an adverse event profile expected for a seriously ill population in the ICU with AKI. The blood flow patency of the SCD was comparable with single-cartridge CRRT modalities. These results suggested a higher dose with a larger membrane surface area, and longer treatment time was safe and potentially more effective.

A US multicenter pilot clinical study was conducted to assess the safety and efficacy of the SCD in ICU patients with acute renal failure and MOF $[39,41]$. This study was undertaken with an FDA-approved investigational device exemption (G090189). A total of 35 patients at 6 clinical sites were enrolled (ClinicalTrials.gov, ID No. NCT01072682). Those 35 patients had an average age of 56 years, an average sequential organ failure assessment score of 11.5 and $28 / 35(80 \%)$ patients were septic. Ninety percent $(31 / 35)$ of the patients were on mechanical ventilation. For the entire patient group, 28-day all-cause mortality was $20 \%$ and 60 -day all-cause mortality was $31 \%$. For the septic patients, 28-day and 60-day all-cause mortality rates were similar to those of the entire patient group outcomes. This compared favorably to historical 
conventional renal replacement therapy mortality rates exceeding 50\% (fig. 3). There were no SCD-treated patients requiring dialysis at 60 days compared to $8 \%$ in the comparative control group.

\section{Conclusion}

Conventional treatment of acute and chronic renal diseases has focused on solute removal. Novel strategies aim to treat the multifactorial disease states of AKI and chronic kidney disease by mitigating inflammation using cell therapy or cell processing approaches. Cell therapy leverages the metabolic and synthetic functions of renal tubule cells to provide gluconeogenesis, ammoniagenesis, metabolism of glutathione, catabolism of important peptide hormones, growth factors, and cytokines critical to multiorgan homeostasis and immunomodulation. A cell processing approach utilizing a promising, new therapeutic device - the SCD - is designed to address the proinflammatory state of kidney dysfunction by binding and deactivating leukocytes.

\section{Acknowledgements}

This work was supported by the US Army Medical Research and Materiel Command, Contract W81XWH-05-2-0010 and W81XWH-10-2-0137, and the Small Business Innovation Research program of the National Institutes of Health, Grants NIDDK R44 DK074289, NIDDK R43 DK080529 and NIDDK R43 DK082050. We acknowledge the provision of human kidneys, used to isolate renal epithelial cells, by the National Disease Research Interchange (NDRI) with support by grant number 5 U42 RR006042 from NIH. The contents of this publication are solely the responsibility of the authors and do not necessarily represent the official views of NIH.

\section{Disclosure Statement}

H.D.H. is a shareholder of Innovative BioTherapies Inc., and CytoPherx Inc. C.J.P. is an employee of Innovative BioTherapies Inc. A.S.Y. is a consultant for CytoPherx Inc.

\section{References}

$\checkmark 1$ Nigam S, Lieberthal W: Acute renal failure. 3. The role of growth factors in the process of renal regeneration and repair. Am J Physiol Renal Physiol 2000;279:F3-F11.

-2 Thadhani R, Pascual M, Bonventre JV: Acute renal failure. N Engl J Med 1996;334:14481460.

-3 Lieberthal W, Nigam SK: Acute renal failure. 2. Experimental models of acute renal failure: imperfect but indispensable. Am J Physiol Renal Physiol 2000;278:F1-F12.

4 Lieberthal W, Nigam SK: Acute renal failure. 1. Relative importance of proximal vs distal tubular injury. Am J Physiol 1998;275:F623F631.

$\checkmark 5$ Breen D, Bihari D: Acute renal failure as a part of multiple organ failure: the slippery slope of critical illness. Kidney Int Suppl 1998;66:S25-S33.

6 US Renal Data System. USRDS 2009 Annual Data Report: Atlas of End-Stage Renal Disease in the United States. Bethesda, National Institute of Diabetes and Digestive and Kidney Diseases, 2009.

7 Humes HD: Bioartificial kidney for full renal replacement therapy. Semin Nephrol 2000;20:71-82.

8 Humes HD, MacKay SM, Funke AJ, Buffington DA: The bioartificial renal tubule assist device to enhance CRRT in acute renal failure. Am J Kidney Dis 1997;30:S28-S31.
$\$ 9$ Lu CY, Hartono J, Senitko M, Chen J: The inflammatory response to ischemic acute kidney injury: a result of the 'right stuff' in the 'wrong place'? Curr Opin Nephrol Hypertens 2007;16:83-89.

10 Okusa MD: The inflammatory cascade in acute ischemic renal failure. Nephron 2002; 90:133-138.

11 Humes HD: Cell therapy: leveraging nature's therapeutic potential. J Am Soc Nephrol 2003; 14:2211-2213.

12 Pino CJ, Humes HD: Stem cell technology for the treatment of acute and chronic renal failure. Transl Res 2010;156:161-168.

13 Humphreys BD, Bonventre JV: The contribution of adult stem cells to renal repair. Nephrol Ther 2007;3:3-10.

14 Bussolati B, Camussi G: Stem cells in acute kidney injury. Contrib Nephrol 2007;156: 250-258.

15 Romagnani P: Toward the identification of a 'renopoietic system'? Stem Cells 2009;27: 2247-2253.

16 Little MH, Bertram JF: Is there such a thing as a renal stem cell? J Am Soc Nephrol 2009; 20:2112-2117.

17 MacKay SM, Funke AJ, Buffington DA, Humes HD: Tissue engineering of a bioartificial renal tubule. ASAIO J 1998;44:179183.
18 Humes HD, Cieslinski DA: Interaction between growth factors and retinoic acid in the induction of kidney tubulogenesis in tissue culture. Exp Cell Res 1992;201:8-15.

19 Humes HD, Krauss JC, Cieslinski DA, Funke AJ: Tubulogenesis from isolated single cells of adult mammalian kidney: clonal analysis with a recombinant retrovirus. Am J Physiol 1996;271:F42-F49.

20 Humes HD, MacKay SM, Funke AJ, Buffington DA: Tissue engineering of a bioartificial renal tubule assist device: in vitro transport and metabolic characteristics. Kidney Int 1999;55:2502-2514

21 Nikolovski J, Gulari E, Humes HD: Design engineering of a bioartificial renal tubule cell therapy device. Cell Transplant 1999;8: 351-364

22 Humes HD, Fissell WH, Weitzel WF: The bioartificial kidney in the treatment of acute renal failure. Kidney Int Suppl 2002;80:121125 .

23 Humes HD, Buffington DA, MacKay SM, Funke AJ, Weitzel WF: Replacement of renal function in uremic animals with a tissue-engineered kidney. Nat Biotechnol 1999;17: 451-455.

24 Humes HD, et al: Cell therapy with a tissueengineered kidney reduces the multiple-organ consequences of septic shock. Crit Care Med 2003;31:2421-2428. 
25 Humes HD, et al: Initial clinical results of the bioartificial kidney containing human cells in ICU patients with acute renal failure. Kidney Int 2004;66:1578-1588.

26 Tumlin J, et al: Efficacy and safety of renal tubule cell therapy for acute renal failure. J Am Soc Nephrol 2008;19:1034-1040.

27 Humes HD, Sobota JT, Ding F, Song JH: A selective cytopheretic inhibitory device to treat the immunological dysregulation of acute and chronic renal failure. Blood Purif 2010;29:183-190.

28 Fahy GM, Wowk B, Wu J: Cryopreservation of complex systems: the missing link in the regenerative medicine supply chain. Rejuvenation Res 2006;9:279-291.

29 Westover AJ, Buffington DA, Humes HD Enhanced propagation of adult human renal epithelial progenitor cells to improve cell sourcing for tissue-engineered therapeutic devices for renal diseases. J Tissue Eng Regen Med 2012;6:589-597.
30 Buffington D, Pino C, Chen L, Westover A, Hageman G, Humes H: Bioartificial renal epithelial cell system (BRECS): a compact, cryopreservable extracorporeal renal replacement device. Cell Medicine 2012;4:33-43.

31 Rojas A, et al: Animal model of a wearable bioartificial kidney using peritoneal dialysis. ASAIO Annu Meet, Dallas, 2009.

32 Song J, et al: Uremic Animal Model of Bioartifical Renal Cell System (BRECS) Using Continuous Flow Peritoneal Dialysis-Based Extracorporeal Circuit. Renal Week 2009, San Diego, Calif., 2009.

33 Tintinger G, Steel HC, Anderson R: Taming the neutrophil: calcium clearance and influx mechanisms as novel targets for pharmacological control. Clin Exp Immunol 2005; 141 : 191-200.

34 Mandeville JT, Maxfield FR: Calcium and signal transduction in granulocytes. Curr Opin Hematol 1996;3:63-70.

35 Ding F, et al: A biomimetic membrane device that modulates the excessive inflammatory response to sepsis. PLoS One 2011;6:e18584.
Pino CJ, Lou L, Smith PL, Ding F, Pagani FD, Buffington DA, Humes HD: A selective cytopheretic inhibitory device for use during cardiopulmonary bypass surgery. Perfusion 2012;27:311-319.

37 Ayub K, Hallett MB: $\mathrm{Ca}^{2+}$ influx shutdown during neutrophil apoptosis: importance and possible mechanism. Immunology 2004; 111:8-12.

38 Ding F, et al: The effects of a novel therapeutic device on acute kidney injury outcomes in the intensive care unit: a pilot study. ASAIO J 2011;57:426-432.

39 Tumlin JA, Chawla L, Tolwani AJ, Mehta R, Dillon J, Finkel K, DaSilva JR, Astor BC, Yevzlin AS, Humes HD: The effect of the selective cytopheretic device on acute kidney injury outcomes in the intensive care unit: a multi-center pilot study. Semin Dial 2012, DOI:10.1111/sdi.12032.

40 Mehta RL, et al: Spectrum of acute renal failure in the intensive care unit: the PICARD experience. Kidney Int 2004;66:1613-1621. 